\title{
Placement of Surgical Mesh
}

National Cancer Institute

\section{Source}

National Cancer Institute. Placement of Surgical Mesh. NCI Thesaurus. Code C129506.

A surgical procedure in which a surgical mesh is implanted, usually for repair of abdominal wall hernias or pelvic organ prolapse. 